\title{
Clinical outcomes of two types of cages used in transforaminal lumbar interbody fusion for the treatment of degenerative lumbar diseases: n-HA/PA66 cages versus PEEK cages
}

\author{
Qian-xing Deng ${ }^{1} \cdot$ Yun-sheng Ou ${ }^{1} \cdot$ Yong Zhu $^{1} \cdot Z_{\text {Zeng-hui Zhao }}{ }^{1} \cdot$ Bo Liu $^{1} \cdot$ \\ Qiu Huang ${ }^{1} \cdot$ Xing Du $^{1} \cdot$ Dian-ming Jiang ${ }^{1}$
}

Received: 20 January 2016/Accepted: 1 April 2016/Published online: 18 April 2016

(c) The Author(s) 2016. This article is published with open access at Springerlink.com

\begin{abstract}
This study reports the clinical effects of nanohydroxyapatite/polyamide66 cages (n-HA/PA66 cages) and compares the clinical outcomes between n-HA/PA66 and polyetheretherketone cages (PEEK cages) for application in transforaminal lumbar interbody fusion (TLIF). A retrospective and case-control study involving 124 patients using n-HA/PA66 cages and 142 patients using PEEK cages was conducted. All patients underwent TLIF and had an average of 2-years of follow-up. The Oswestry Disability Index and Visual Analog Scale were selected to assess the pain of low back and leg, as well as neurological status. The intervertebral space height and segmental angle were also measured to estimate the radiological changes. At the 1-year and final follow-ups, the fusion and subsidence rates were evaluated. There was no significant difference between the two groups regarding clinical and radiological results. At the final follow-up, the bony fusion rate was 92.45 and $91.57 \%$ for the n-HA/PA66 and PEEK groups, respectively, and the subsidence rate was 7.55 and $8.99 \%$, respectively. The study indicated that both n-HA/ PA66 and PEEK cages could promote effective clinical and radiographic outcomes when used to treat degenerative lumbar diseases. The high fusion and low subsidence rates revealed that n-HA/PA66 cages could be an alternative ideal choice as the same to PEEK cages for lumbar reconstruction after TLIF.
\end{abstract}

Yun-sheng Ou

ouyunsheng2001@163.com

1 Department of Orthopedics, The First Affiliated Hospital of Chongqing Medical University, YouYi Road 1\#, YuZhong District, Chongqing 400016, People's Republic of China

\section{Introduction}

TLIF, which was described by Harms and Rolinger [1], is widely applied for the treatment of degenerative lumbar diseases with favorable outcomes [2-8]. One of the biggest advantages of TLIF is that it decreases the postoperative neurological deficit by reducing excessive neural tissue and dural sac retraction compared with posterior lumbar interbody fusion [9, 10]. Other advantages include avoiding potential complications associated with anterior lumbar interbody fusion, shorter hospital stays and lower costs compared with anterior combined with posterior approaches [11]. In addition, TLIF theoretically offers a lower risk of segmental instability because of the preservation of posterior lamina arch and posterior longitudinal ligament complex [9]. Typical indications for TLIF are degenerative or isthmus spondylolisthesis, degenerative disc disease, lumbar stenosis, lumbar disc herniation and recurrent lumbar disc herniation [9, 12].

Alternative materials for interbody fusion include autograft iliac crest, allograft bone, carbon fiber cages, titanium mesh cages, PEEK and n-HA/PA66 cages [4, 13-16]. Auto-graft iliac crest has been considered the "gold standard" for anterior column reconstruction, but there are some donor-site complications [4]. PEEK cages are radiolucent and have an elastic modulus similar to native bone [17]. PEEK cages augmented by pedicle screws have been shown to promote lumbar interbody fusion and to provide excellent clinical outcomes [3, 18]. The n-HA/PA66 cages are hollow bullets consisting of n-HA/PA66 composite, which simulates the constituent form of native bone [1924]. In recent years, n-HA/PA66 cages filled with autograft bone have been reported to treat cervical spondylosis, cervical spondylotic myelopathy and thoraco-lumbar fractures with satisfactory clinical outcomes [15, 16, 25, 26]. 
However, to the best of our knowledge, there is a lack of literatures reporting the clinical application of n-HA/PA66 cages for the treatment of degenerative lumbar diseases, and there are little articles comparing the clinical efficacy of n-HA/PA66 cages and PEEK cages. Furthermore, the reconstruction and bony fusion of lumbar spine after discectomy remains challenge. The present retrospective study aimed to compare the clinical outcomes of n-HA/PA66 and PEEK cages used in TLIF. Thus, we make a hypothesis that n-HA/PA66 cages can lead to favorable clinical efficacy, and that both clinical and iconographic outcomes of n-HA/ PA66 cages are corresponding to that of PEEK cages.

\section{Materials and methods}

\subsection{Patients}

The primary study subjects were patients who were diagnosed with degenerative or isthmus spondylolisthesis, degenerative disc disease, lumbar stenosis, lumbar disc herniation or recurrent lumbar disc herniation between August 2010 and December 2013. We excluded patients with lumbar tuberculosis, tumor or infection or trauma or who lacked sufficient clinical data. The patients were retrospectively divided into two groups based on cage types.

\subsection{Interbody cages}

The n-HA/PA66 cages were designed and fabricated by the Institution of Materials Science and Technology, Sichuan University, and our department (Fig. 1). The PEEK cages were from the Shandong We-go Orthopedic Group Medical Polymer CO., Ltd. Shandong, China (Fig. 2).

\subsection{Surgical procedures}

All patients underwent preoperative examinations, including static and lateral flexion/extension radiographs and computed tomography scan. Neurogenic claudication, low back pain, and radicular symptoms were investigated with magnetic resonance imaging. TLIF was conducted as descriped by Meyer et al. [9]. In this protocol, we assumed that hypertrophic osteophytes surrounding the lateral recess and the ligamentum flavum were removed in every case to ensure that the dura mater was widely exposed and that the nerve root was released. The adjacent cartilage endplates were removed as fully as possible, but the bony endplates were preserved.

All patients were instructed to wear a lumbar brace for a period of approximately 12 weeks and had 3-, 6- and 12-month follow-ups, as well as a final follow-up. Static and lateral flexion/extension radiographs were used to assess the instruments, stability, lumbar curvature and discspace height of the fused segments and bony fusion status. If necessary, CT scan was taken for further evaluation.

\subsection{Outcome measurements}

The surgery time, blood loss, and perioperative complications were recorded. The ODI and VAS were applied to evaluate the pain of low back and leg, as well as neurological status at preoperative, 12-month and final follow-up time points. At the preoperative, 1-week, 3-, 6-, and 12-month and final follow-up time points, we measured the intervertebral
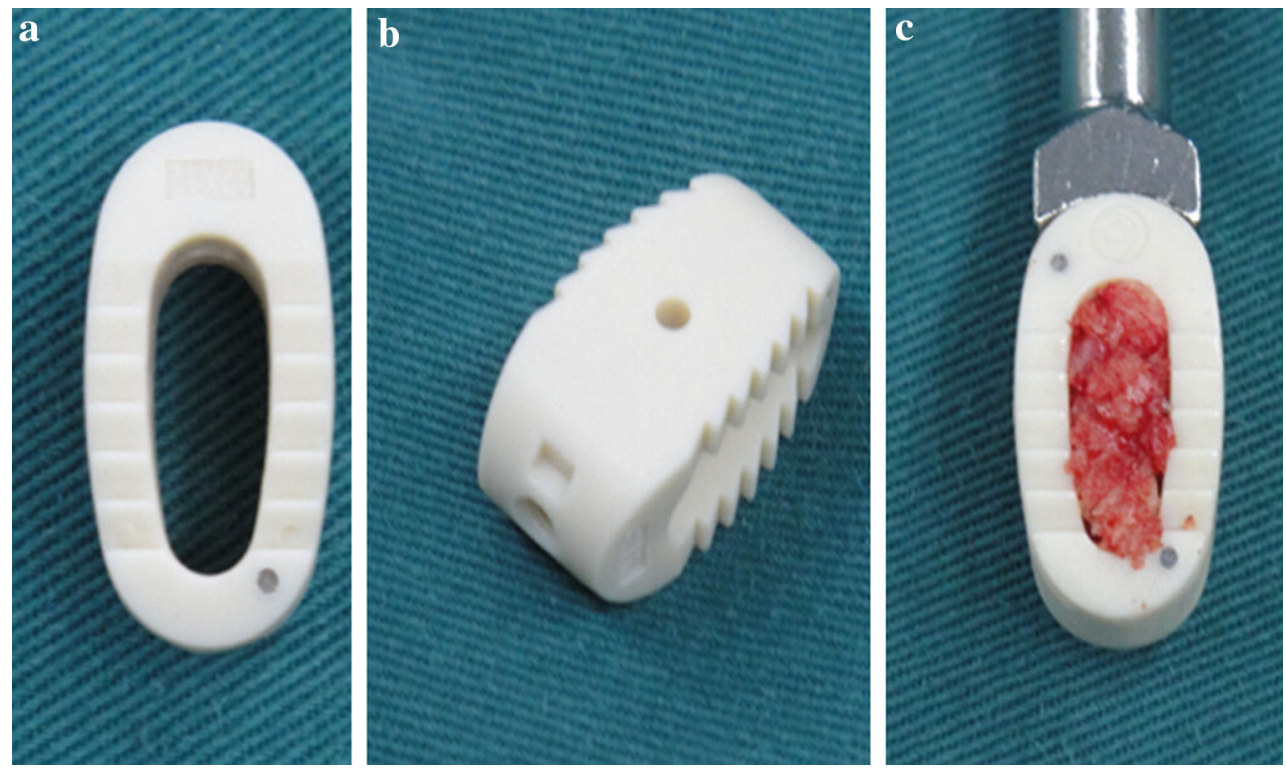

Fig. 1 Photos of n-HA/PA66 cages: superior (a) and lateral (b) views and packed with osseous granula (c) 

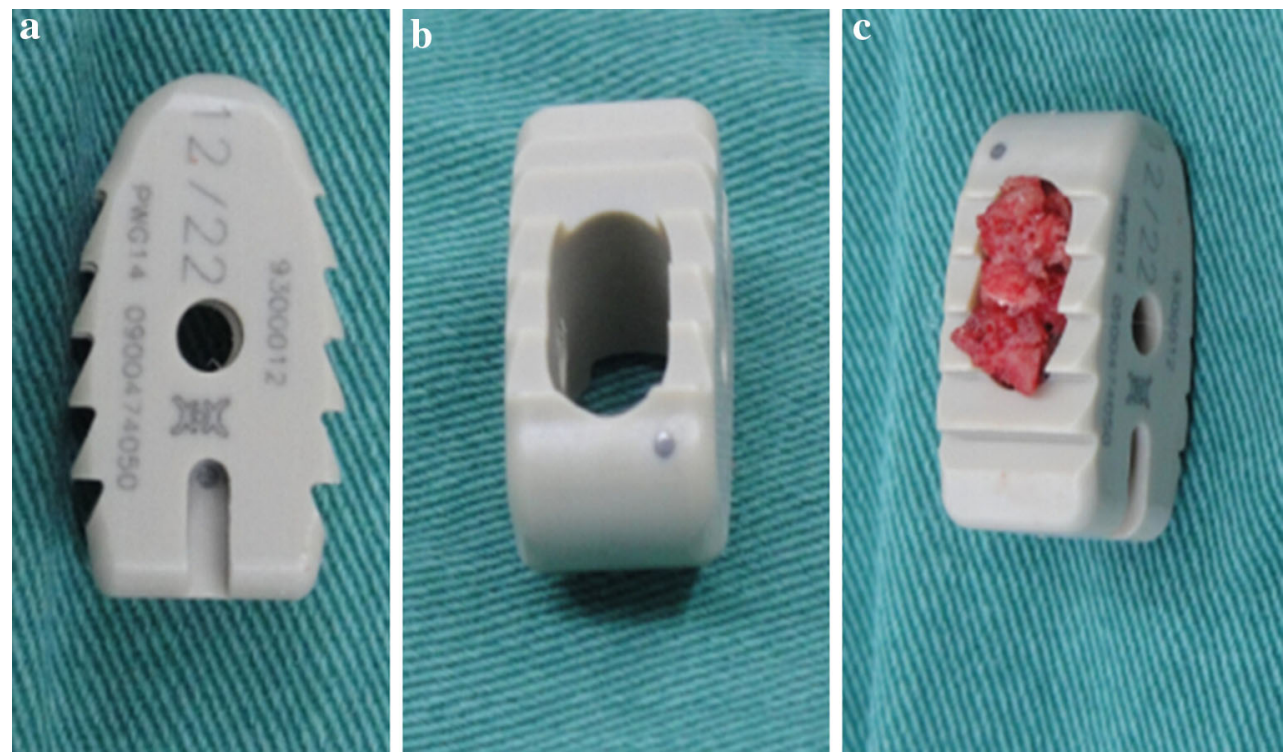

Fig. 2 Photos of PEEK cages: superior (a) and lateral (b) views and packed with osseous granula (c)

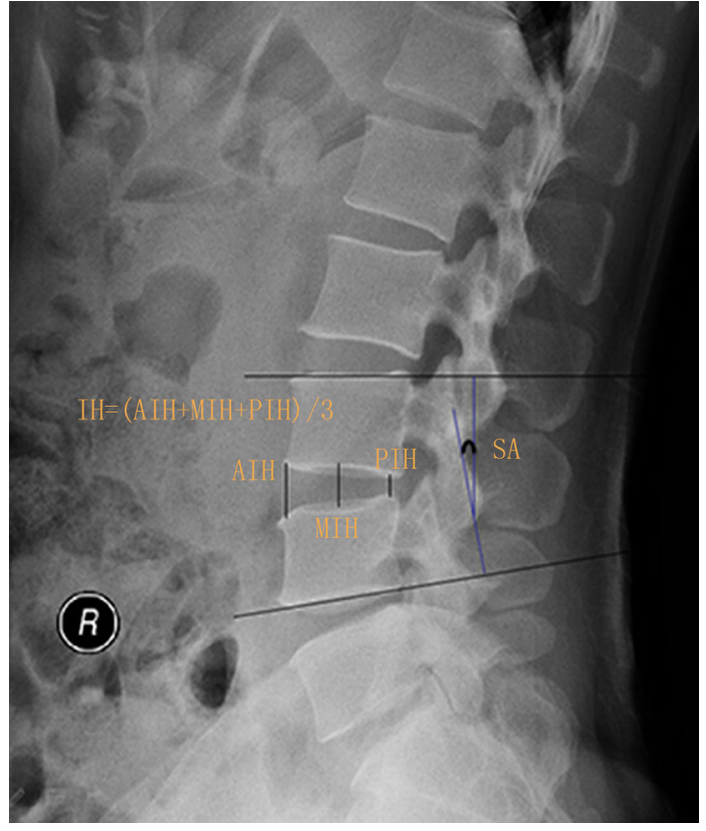

Fig. 3 Methods to measure intervertebral space height and segmental angle. $\mathrm{IH}=(\mathrm{AIH}+\mathrm{MIH}+\mathrm{PIH}) / 3 . A I H$ anterior intervertebral space height; $M I H$ middle intervertebral space height; $P I H$ posterior intervertebral space height. SA, between the superior endplate of upper vertebral and inferior endplate of lower vertebral of fused segment on neutral lateral lumbar plain film

space height (IH, Fig. 3) and segmental angle (SA, Fig. 3) using Carestream software (versions 10.0, Carestream Health, Eastman Kodak, Inc. Rochester, NY, USA). The loss of IH and SA was defined as the D-value between final and 1-week postoperative follow-ups. The cage subsidence was defined as any loss of IH more than $3 \mathrm{~mm}$ [27].
Bony fusion was identified by the following: the presence of trabeculation and bone bridging between cages and adjacent endplates, the absence of greater than $3 \mathrm{~mm}$ translational motion and more than $5^{\circ}$ angular motion upon flexion/extension radiographs in the fused segments and the absence of a radiolucent gap between the cages and endplates [28]. If the surgeons were uncertain, three-dimensional computed tomography scans were taken to verify the fusion status by observing the trabeculation between the autogenous cancellous graft and adjacent endplates.

\subsection{Statistical analyses}

All statistical analysis was performed using the Statistical Package for the Social Sciences version 17.0 (SPSS, Chicago, IL, USA). Quantitative data are presented as the mean \pm standard deviation. Repeated measures ANOVA was used for statistical analyses of differences in mean values, and the Chi-squared test was used for categorical data between groups. The independent $t$ test was applied to compare the clinical and radiological data of two cages. Significant difference was accepted at $P<0.05$.

\section{Results}

\subsection{Patient demographics}

A total of 266 patients with an average $24.24 \pm 8.97$ months of follow-up (range 12-47 months) were included in this study. Of these, 124 patients underwent TLIF with an n-HA/ PA66 cage (Fig. 4) and 142 patients underwent TLIF with a 

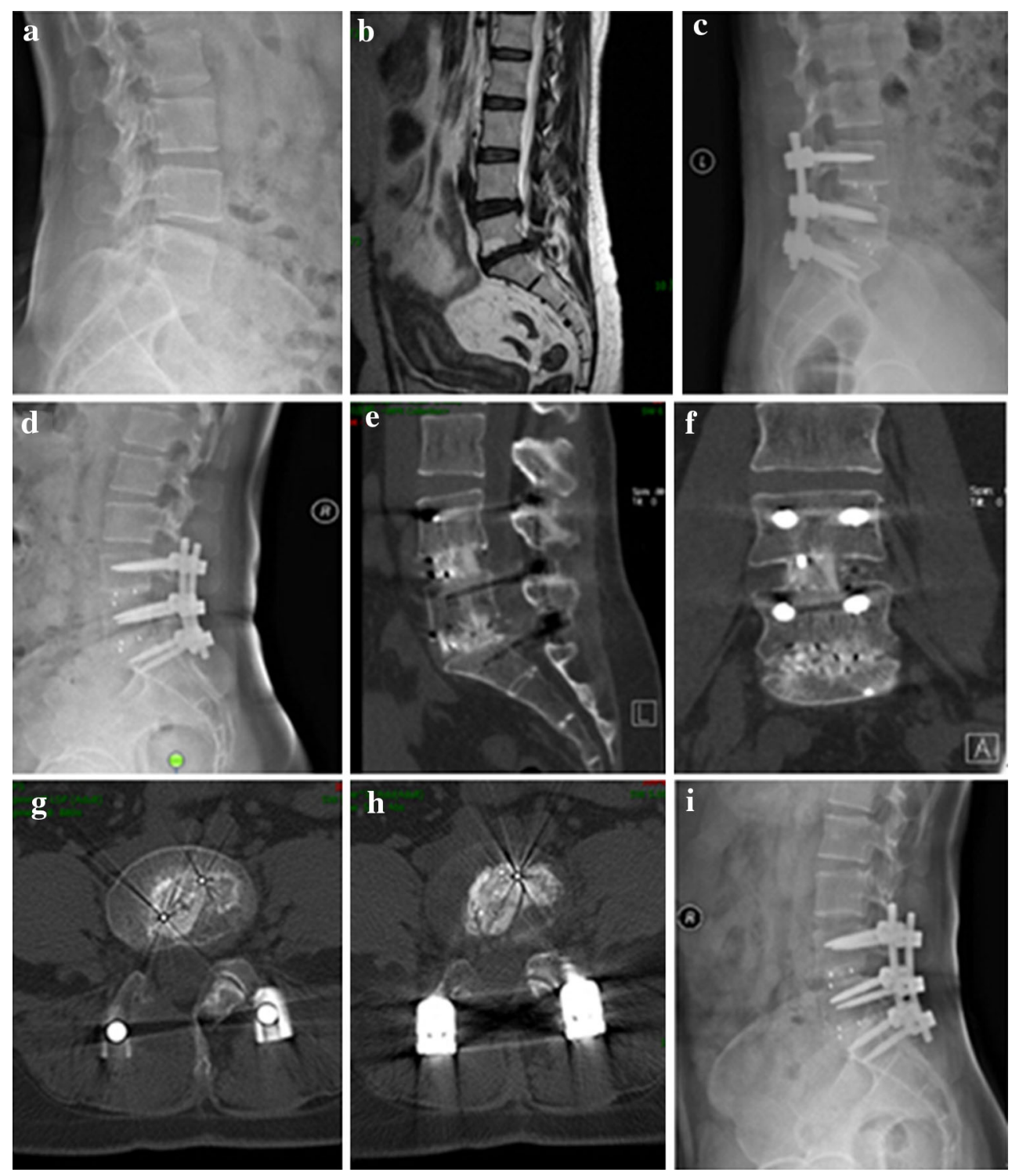

Fig. 4 A 42-year-old female who underwent 2-level TLIF with n-HA/PA66 cages for lumbar reconstruction. The preoperative lumbar radiographs $(\mathbf{a}, \mathbf{b})$. The 1-week postoperative and 3-month follow-up radiographs $(\mathbf{c}, \mathbf{d})$. The $\mathrm{CT}$ or $3 \mathrm{D}-\mathrm{CT}$ scan $(\mathbf{e}, \mathbf{f}, \mathbf{g}, \mathbf{h})$ shows that the

PEEK cage (Fig. 5). The demographics of the patients were shown in Table 1. No significant differences were detected in gender, age, course of disease, surgery time, blood loss, or perioperative complications between the n-HA/PA66 and PEEK cage groups.

\subsection{Radiological outcomes}

The IH was improved in the n-HA/PA66 group from $9.44 \pm 2.16 \mathrm{~mm}$ preoperative to $12.62 \pm 1.58 \mathrm{~mm}$ at 1-week postoperative and in the PEEK group from $9.28 \pm 2.14 \mathrm{~mm}$ preoperative to $12.51 \pm 1.72 \mathrm{~mm}$ at 1-week postoperative. The average correction of the $\mathrm{IH}$ autogenous bone granules fill the cages and achieve bony fusion with adjacent endplates by the 10-month follow-up. A lateral radiograph (i) at the final follow-up shows satisfactory bony fusion and no obvious migration, radiolucent gap or subsidence

was $3.18 \pm 1.73 \mathrm{~mm}$ in the $\mathrm{n}$-HA/PA66 group, and the mean loss of the IH was $1.65 \pm 0.87 \mathrm{~mm}$. There were no significant differences between the two groups for all of the above parameters at any time point observed $(P>0.05$, Table 2). Regarding the SA, it also did not differ significantly $(P>0.05)$ except in the loss of SA $(P=0.044$, Table 2).

At the 1-year follow-up, $87.42 \%$ of patients in the n-HA/PA66 group and $87.08 \%$ of patients in the PEEK group showed bony fusion. At the final follow-up, the bony fusion rate was 92.45 and $91.57 \%$ in the n-HA/PA66 and PEEK groups, respectively. Three months after operation, the cage subsidence was $3.77 \%$ in the n-HA/PA66 group 

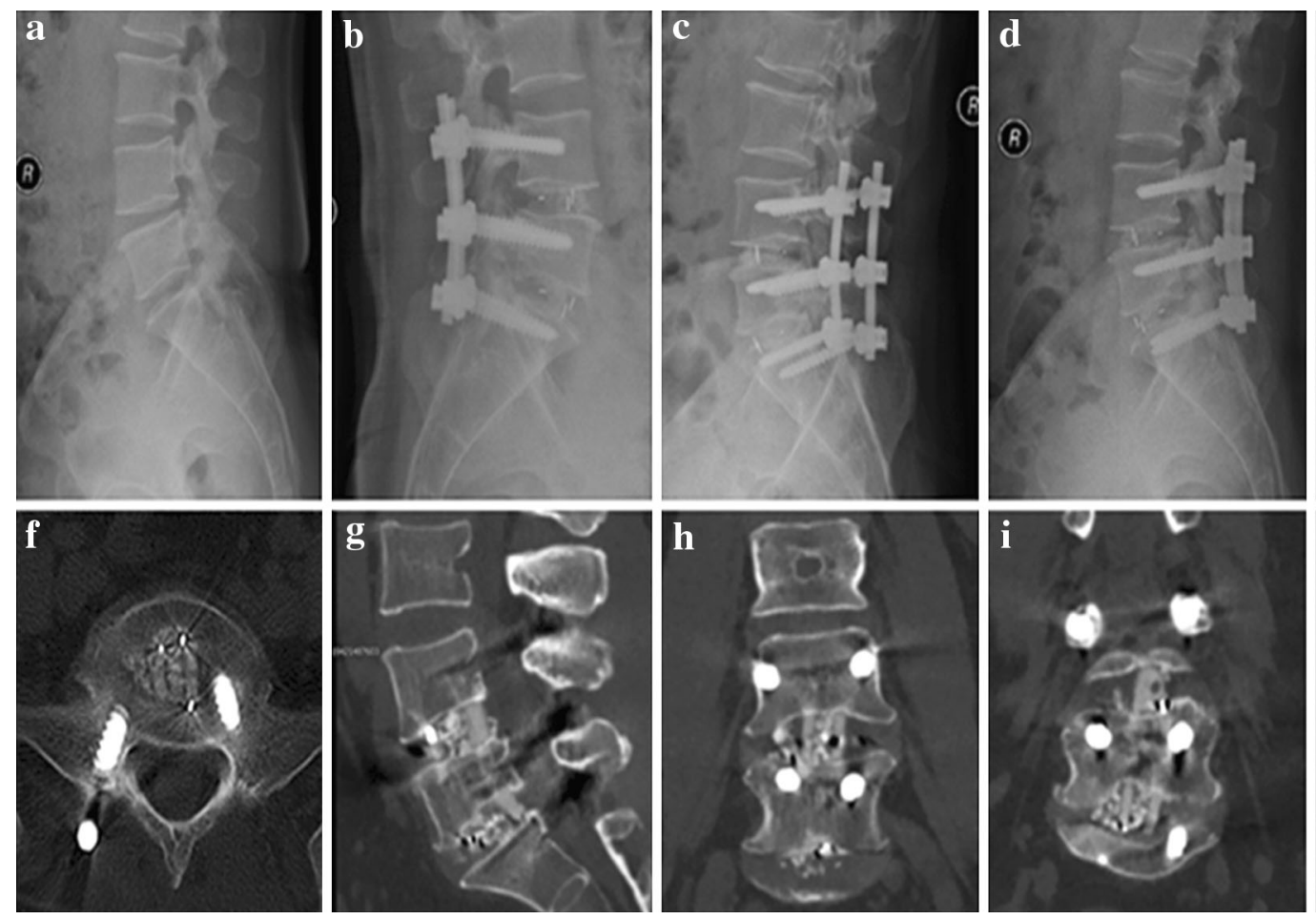

Fig. 5 A 58-year-old male who underwent 2-level TLIF with PEEK cages for lumbar reconstruction. The preoperative radiograph (a). The 1-week postoperative and 3-month and 6-month follow-up radiographs $(\mathbf{b}, \mathbf{c}, \mathbf{d})$. The CT or $3 \mathrm{D}-\mathrm{CT} \operatorname{scan}(\mathbf{e}, \mathbf{f}, \mathbf{g}, \mathbf{h}, \mathbf{i})$ shows that the autogenous bone granules fill the cages and achieve bony fusion with adjacent endplates by the 12-month follow-up. A lateral radiograph (j) at the final follow-up shows satisfactory bony fusion and no obvious migration, radiolucent gap or subsidence
Table 1 The demographic and clinical data of patients

\begin{tabular}{llll}
\hline Parameters & n-HA/PA66 Cage $(\mathrm{n}=124)$ & PEEK Cage $(\mathrm{n}=142)$ & $P$ \\
\hline Male/female & $61 / 63$ & $60 / 82$ & 0.257 \\
Age & $53.28 \pm 12.51$ & $53.65 \pm 14.43$ & 0.823 \\
Course of disease & $43.74 \pm 60.95$ & $44.68 \pm 61.50$ & 0.901 \\
Diagnosis & & & \\
LDH & 79 & 90 & \\
LS & 34 & 35 & \\
LSS & 6 & 6 & 0.332 \\
Revision & 4 & 10 & 0.188 \\
LDS & 1 & 1 & 0.804 \\
Surgery time & $169.31 \pm 34.25$ & $164.82 \pm 40.22$ & 0.619 \\
Blood loss & $268.79 \pm 193.52$ & $236.69 \pm 201.63$ & \\
Perioperative complication & $17 / 124(13.71 \%)$ & $18 / 142(12.68 \%)$ & \\
Follow up & $23.94 \pm 9.17$ & $24.49 \pm 8.81$ & \\
Segments & & & $108 / 32 / 2$ \\
1-L,2-L,3-L & $89 / 35 / 0$ & $4 / 1 / 21 / 89 / 63$ & \\
L1/2,L2/3,L3/4,L4/5,L5/S1 & $0 / 2 / 16 / 86 / 55$ & & \\
\hline
\end{tabular}

$L D H$ lumbar disc herniation, $L S$ lumbar spondylolisthesis, $L S S$ lumbar spinal stenosis, $L D S$ lumbar degenerative scoliosis

and $2.25 \%$ in the PEEK group. At 6 months as well as at 1 year and the final follow-up, there were no significant differences in the bony fusion and cage subsidence rates $(P>0.05$, Table 2).

\subsection{Clinical outcomes}

The preoperative ODI and VAS scores did not differ between the n-HA/PA66 and PEEK groups. Upon follow 
Table 2 SA, IH, and fusion and subsidence rates at various time points (Mean \pm SD)

\begin{tabular}{|c|c|c|c|}
\hline Parameters & n-HA/PA66 Cage $(\mathrm{n}=159)$ & PEEK Cage $(\mathrm{n}=178)$ & $P$ \\
\hline \multicolumn{4}{|l|}{$\mathrm{SA}\left({ }^{\circ}\right)$} \\
\hline Pre-O & $17.94 \pm 8.49$ & $16.91 \pm 8.53$ & 0.267 \\
\hline 1-w Post-O & $18.50 \pm 6.09$ & $18.54 \pm 6.71$ & 0.954 \\
\hline 3-m Post-O & $18.24 \pm 6.00$ & $18.10 \pm 6.62$ & 0.839 \\
\hline 6-m Post-O & $17.65 \pm 5.64$ & $17.47 \pm 6.42$ & 0.775 \\
\hline 1-y Post-O & $17.24 \pm 5.66$ & $16.91 \pm 6.27$ & 0.605 \\
\hline Fin-foll-up & $16.82 \pm 5.61$ & $16.38 \pm 6.11$ & 0.495 \\
\hline Correction & $0.55 \pm 5.89$ & $1.63 \pm 4.91$ & 0.072 \\
\hline Loss & $1.68 \pm 2.07$ & $2.16 \pm 2.26$ & 0.044 \\
\hline \multicolumn{4}{|l|}{ IH (mm) } \\
\hline Pre-O & $9.44 \pm 2.16$ & $9.28 \pm 2.14$ & 0.490 \\
\hline 1-w Post-O & $12.62 \pm 1.58$ & $12.51 \pm 1.72$ & 0.536 \\
\hline 3-m Post-O & $11.95 \pm 1.48$ & $11.70 \pm 1.47$ & 0.114 \\
\hline 6-m Post-O & $11.55 \pm 1.41$ & $11.32 \pm 1.36$ & 0.141 \\
\hline 1-y Post-O & $11.27 \pm 1.32$ & $11.07 \pm 1.39$ & 0.177 \\
\hline Fin-foll-up & $10.97 \pm 1.26$ & $10.86 \pm 1.37$ & 0.453 \\
\hline Correction & $3.18 \pm 1.73$ & $3.23 \pm 1.72$ & 0.791 \\
\hline Loss & $1.65 \pm 0.87$ & $1.65 \pm 0.97$ & 0.966 \\
\hline \multicolumn{4}{|c|}{ Fusion rate $(\%)$} \\
\hline 1-y Post-O & 139/159 (87.42 \%) & 155/178 (87.08 \%) & 0.925 \\
\hline Fin-foll-up & $147 / 159(92.45 \%)$ & 163/178 (91.57\%) & 0.766 \\
\hline \multicolumn{4}{|c|}{ Subsidence rate $(\%)$} \\
\hline 3-m Post-O & 6/159 (3.77\%) & $4 / 178(2.25 \%)$ & 0.526 \\
\hline 6-m Post-O & $9 / 159(5.66 \%)$ & $6 / 178(3.37 \%)$ & 0.309 \\
\hline 1-y Post-O & $11 / 159(6.92 \%)$ & $10 / 178(5.62 \%)$ & 0.622 \\
\hline Fin-foll-up & $12 / 159(7.55 \%)$ & $16 / 178(8.99 \%)$ & 0.632 \\
\hline
\end{tabular}

Pre-O pre-operation, Post-O post-operation, Fin-foll-up final follow-up up, VAS scores had improved significantly for both groups, but no significant differences were found between the n-HA/PA66 and PEEK groups $(P>0.05$, Table 3$)$. The ODI was also similar between the two types of cage groups during follow up $(P>0.05$, Table 3$)$.

\section{Discussion}

In recent years, TLIF for the treatment of lumbar degenerative diseases has become a widely used surgery. The most important factors are the thorough decompression of nerve and/or cauda equina and the bony fusion of the anatomic anterior column [29]. However, in reconstruction and bony fusion, it is important to avoid complications, such as the failure of internal fixation and cage migration, which is challenging work for orthopedic surgeons [30].

Currently, PEEK cages have been widely used. Lee et al. [18] evaluated the fusion rate of a morselized local bone graft in PEEK cages. They obtained an $86.7 \%$ fusion rate at 6-months and a $90.0 \%$ fusion rate at 12 -months
Table 3 VAS and ODI at pre-o and post-o (mean \pm SD)

\begin{tabular}{lccl}
\hline Parameters & n-HA/PA66 Cage & PEEK Cage & $P$ \\
\hline VAS (points) & & & \\
Pre-O & $6.02 \pm 1.20$ & $6.17 \pm 1.38$ & 0.338 \\
1-y Post-O & $2.31 \pm 0.85$ & $2.25 \pm 0.87$ & 0.617 \\
Fin-foll-up & $1.56 \pm 0.87$ & $1.58 \pm 0.89$ & 0.853 \\
ODI (\%) & & & \\
Pre-O & $50.56 \pm 6.41$ & $51.00 \pm 6.47$ & 0.583 \\
1-y Post-O & $26.37 \pm 5.94$ & $26.51 \pm 5.88$ & 0.851 \\
Fin-foll-up & $14.69 \pm 4.13$ & $14.61 \pm 4.08$ & 0.862 \\
\hline
\end{tabular}

follow-up. They believed that 1-year post-operation was a better time point for observing bony fusion. However, the K-ODI, SF-36 and VAS values were similar after surgery. Whether there was a relationship between fusion rate and clinical outcomes remains unknown [18]. Schomacher et al. [31] reported the application of TMCs and PEEK cages for the treatment of pyogenic spondylodiscitis. The solid bony fusion rate was $90.5 \%$ in the PEEK group and 
$100 \%$ in the TMCs group, but the difference was not significant. Nemoto et al. [8] compared TMCs and PPEK cages in their study and found bony fusion rates of 96 and $64 \%$ at 12-months and 100 and $76 \%$ at 2-years after surgery, respectively. They concluded that there was no demonstrable superiority of PEEK cages over TMCs in regards to bony fusion. Additionally, they found unfavorable vertebral osteolysis in PEEK cages, which may lead to nonunion, and suggested that the improvement in the biocompatibility of PEEK cages was necessary to increase fusion rates [8].

TMCs used for spinal reconstruction have been reported with many disadvantages. For example, Jang et al. [14] found that cage subsidence occurred in $93.3 \%$ of patients after anterior cervical corpectomy and reconstruction, although the fusion rate was $100 \%$. They thought that cage subsidence could make up for the advantages of TMCs, such as restoration and maintenance of $\mathrm{IH}$, enlargement of the stenotic neural foramen and immediate stabilization of operative segments. Whether there was a relationship between cage subsidence and clinical effects remains unclear. Yang et al. [15] compared TMCs with n-HA/PA66 cages for onelevel anterior cervical corpectomy and fusion (ACCF) and observed that the fusion rate of the n-HA/PA66 group was higher than TMCs at 1-year follow-up but that the finial fusion rate was similar. Cage subsidence was significantly lower in the n-HA/PA66 group than in TMCs. The VAS and JOA in the TMCs group were worse than in the n-HA/PA66 group. Zhang et al. [16] reported another comparison between TMCs and n-HA/PA66 cages. According to their study, the fusion rate in the n-HA/PA66 group was higher at the one-year follow-up than the TMCs group for both 1-level and 2-level ACCF, and the cage subsidence was significantly higher in the TMCs group for the 1-level ACCF. Additionally, the difference was significant for the 2-level ACCF between the TMCs and n-HA/PA66 groups.

The n-HA/PA66 cages are made by the Institution of Materials Science and Technology, Sichuan University, and our department. The application was conducted in reconstruction of spine, especially cervical spine. Little articles were used to compare the efficacy of n-HA/PA66 cages with PEEK cages when treating degenerative lumbar diseases. In the present study, we found that the preoperative IH and SA were similar in both the n-HA/PA66 and PEEK groups. Additionally, there were no significant differences in IH and SA at 1-week, 3-, 6-month, 1-year and the final follow-up between the two groups. The correction and loss of IH for the two groups did not differ; neither did the correction of SA. However, the loss of SA was different. In our series, the mean correction of SA in the n-HA/PA66 group was $0.55 \pm 5.89^{\circ}$, while the mean correction of SA was greater in the PEEK group. There may be a tendency toward "the more correction, the more loss", as reported by Rousseau et al. [3]. We thought that loss in lordosis might be related to increased postoperative lordosis and a tendency to recover the initial spinal sagittal balance. However, the fusion and subsidence rates were not affected in our study. Some investigators hold that the loss of IH and SA should be considered a normal and expected result, as slight loss of $\mathrm{IH}$ and SA did not affect clinical outcomes [32, 33]. However, others believed that loss of IH and SA were related to the stability and sagittal sequence of the spine and decompression of nerve root and/or cauda equina, especially excessive subsidence [5, 6]. In our report, the VAS and ODI scores increased after operation and did not show a difference between the two groups at any of the time points examined. No failure of internal instrument or pseudarthrosis or obvious vertebral osteolysis was observed during follow-up.

Cage subsidences are influenced by the lower fused segment, cage position, number of fused segments, cage size, amount of morselized bone, end-plate manipulation and the material characteristics of the cage [3, 5-7]. Regarding the cage material characteristics, both n-HA/ PA66 and PEEK cages have a low Young's modulus, similar to natural bone, resulting in lower stress-shielding compared with TMCs [15-17]. In our study, the n-HA/PA66 cage is made from a composite of nano-hydroxyapatite and polyamide66. Hydroxyapatite, a component of natural bone, is nanocrystallized and then well-distributed into polyamide. The composite possesses both the mechanical strength of Hydroxyapatite and the elastic properties of polyamide66. Studies have demonstrated the biocompatibility, safety, osteoconduction and biomechanical stability of n-HA/PA66 fairly well [19-24]. Additionally, the cage shape is characterized by a wide rim with several shallow recesses to prevent cage migration and subsidence via increasing the friction between the cage and end-plate and dispersing pressure on the cage surface. Animal experiments demonstrated that when implanted, the cage can release $\mathrm{Ca}^{2+}$ and $\mathrm{PO}_{4}{ }^{3-}$ from its surface, which gradually forms a crystal layer on the cage surface that bridges the graft and implant bed to provide a trestle for osteogenesis [34]. In addition, the $2 \mathrm{~mm}$ holes in the cage walls and grooves theoretically allows the invasion of vessels, growth factors, osteogenic factors and bone morphogenetic proteins to promote bone healing and bony fusion. In the current study, $92.45 \%$ of patients showed bony fusion at the final follow-up. Only $7.55 \%$ of patients suffered from cage subsidence in the n-HA/PA66 group, which was similar to the PEEK group. Meanwhile, the VAS and ODI scores were obviously improved. Considering the high fusion and low subsidence rates similar to other reports $[15,16,25,26]$, we suggest that n-HA/PA66 cages are comparable to PEEK cages as ideal implants for application in TLIF. 
Since the application of n-HA/PA66 cages for lumbar spine treatment in 2010, surgeons are expected to gain experience in the following procedures: (1) measure the preoperative IH and choose a suitable cage size; (2) avoid excessive distraction of the intervertebral space, generally 3-4 $\mathrm{mm}$, or the immediate postoperative $\mathrm{IH}$, generally $12-14 \mathrm{~mm}$; (3) maintain the bone end-plate so that it is not broken, while cleaning up the cartilage end-plate completely; (4) ensure sufficient osseous granula to fill the cage; and (5) use a correction of SA that is not great but should correspond to the previous sagittal balance.

Several limitations remain for the present report. Firstly, this was only a case-control and retrospective analysis for the use of n-HA/PA66 and PEEK cages in TLIF. A prospective study is necessary to further confirm the differences observed. Secondly, we selected patients with different diagnoses and segments, which might have some influence on the results. Thus, a layering study should be conducted.

\section{Conclusions}

This case-control and retrospective study demonstrated that the use of both n-HA/PA66 and PEEK cages can promote effective clinical and radiographic outcomes in the treatment of lumbar degenerative diseases with an average 2-year follow-up. The high fusion and low subsidence rates demonstrated that the n-HA/PA66 cage is an alternative ideal substitute material comparable to PEEK cages for lumbar reconstruction after TLIF.

Acknowledgments This study was approved by the Institutional Review Board of the First Affiliated Hospital of Chongqing Medical University which also waived the requirement for patient consent because this study was retrospective, and the data were analyzed anonymously.

\section{Compliance with ethical standards}

Conflict of interest The authors received no specific funding for this work and have declared that no competing interests exist.

Open Access This article is distributed under the terms of the Creative Commons Attribution 4.0 International License (http://creative commons.org/licenses/by/4.0/), which permits unrestricted use, distribution, and reproduction in any medium, provided you give appropriate credit to the original author(s) and the source, provide a link to the Creative Commons license, and indicate if changes were made.

\section{References}

1. Harms J, Rolinger H. A one-stage procedure in operative treatment of spondylolisthesis: dorsal traction-reposition and anterior fusion. Z Orthop Ihre Grenzgeb. 1982;120(3):343-7.
2. Elgafy H, Olson D, Liu J, et al. Effectiveness and safety of transforaminal lumbar interbody fusion in patients with previous laminectomy. Eur Spine J. 2015;24(4):810-6.

3. Rousseau MA, Lazennec JY, Saillant G. Circumferential arthrodesis using PEEK cages at the lumbar Spine. J Spinal Disord Tech. 2007;20(4):278-81.

4. Rihn JA, Patel R, Makda J, et al. Complications associated with single-level transforaminal lumbar interbody fusion. Spine J. 2009;9(8):623-9.

5. Le TV, Baaj AA, Dakwar E, et al. Subsidence of polyetheretherketone intervertebral cages in minimally invasive lateral retroperitoneal transpsoas lumbar interbody fusion. Spine (Phila Pa 1976). 2012;37(14):1268-73.

6. Kim MC, Chung HT, Cho JL, et al. Subsidence of polyetheretherketone cage after minimally invasive transforaminal lumbar interbody fusion. J Spinal Disord Tech. 2013;26(2):87-92.

7. Stein IC, Than KD, Chen KS, et al. Failure of a polyether-etherketone expandable interbody cage following transforaminal lumbar interbody fusion. Eur Spine J. 2015;24(Suppl4):S555-9.

8. Nemoto O, Asazuma T, Yato Y, et al. Comparison of fusion rates following transforaminal lumbar interbody fusion using polyetheretherketone cages or titanium cages with transpedicular instrumentation. Eur Spine J. 2014;23(10):2150-5.

9. Meyer SA, Wu JC, Mummaneni PV. Mini-open and minimally invasive transforaminal lumbar interbody fusion: technique review. Semin Spine Surg. 2011. doi:10.1053/j.semss.2010.12.004.

10. Zhang Q, Yuan Z, Zhou M, et al. A comparison of posterior lumbar interbody fusion and transforaminal lumbar interbody fusion: a literature review and meta-analysis. BMC Musculoskelet Disord. 2014. doi:10.1186/1471-2474-15-367.

11. Ambati DV, Wright EK Jr, Lehman RA Jr, et al. Bilateral pedicle screw fixation provides superior biomechanical stability in transforaminal lumbar interbody fusion: a finite element study. Spine J. 2015;15(8):1812-22.

12. Singh K, Vaccaro AR. Treatment of lumbar instability: transforaminal lumbar interbody fusion. Semin Spine Surg. 2005;17: 259-66. doi:10.1053/j.semss.2005.10.005.

13. Bartels RH, Donk RD, Feuth T. Subsidence of stand-alone cervical carbon fiber cages. Neurosurgery. 2006;58(3):502-8.

14. Jang JW, Lee JK, Lee JH, et al. Effect of posterior subsidence on cervical alignment after anterior cervical corpectomy and reconstruction using titanium mesh cages in degenerative cervical disease. J Clin Neurosci. 2014;21(10):1779-85.

15. Yang X, Chen Q, Liu L, et al. Comparison of anterior cervical fusion by titanium mesh cage versus nano-hydroxyapatite/polyamide cage following single-level corpectomy. Int Orthop. 2013; 37(12):2421-7.

16. Zhang Y, Quan Z, Zhao Z, et al. Evaluation of anterior cervical reconstruction with titanium mesh cages versus nano-hydroxyapatite/polyamide66 cages after 1- or 2-Level corpectomy for multilevel cervical spondylotic myelopathy: a retrospective study of 117 patients. PLoS ONE. 2014;9(5):e96265.

17. Kersten RF, van Gaalen SM, de Gast A, et al. Polyetheretherketone (PEEK) cages in cervical applications: a systematic review. Spine J. 2015;15(6):1446-60.

18. Lee JH, Lee JH, Park JW, et al. Fusion rates of a morselized local bone graft in polyetheretherketone cages in posterior lumbar interbody fusion by quantitative analysis using consecutive three-dimensional computed tomography scans. Spine J. 2011;11(7):647-53.

19. Huang $\mathrm{D}$, Zuo Y, Li J, et al. Bioactive composite gradient coatings of nano-hydroxyapatite/polyamide66 fabricated on polyamide66 substrates. J R Soc Interface. 2012;9(72):1450-7.

20. Guo J, Meng Z, Chen G, et al. Restoration of critical-size defects in the rabbit mandible using porous nanohydroxyapatite-polyamide scaffolds. Tissue Eng Part A. 2012;18(11-12):1239-52. 
21. Wang H, Li Y, Zuo Y, et al. Biocompatibility and osteogenesis of biomimetic nano-hydroxyapatite/polyamide composite scaffolds for bone tissue engineering. Biomaterials. 2007;28(22):3338-48.

22. Wei G, Ma PX. Structure and properties of nano-hydroxyapatite/ polymer composite scaffolds for bone tissue engineering. Biomaterials. 2004;25(19):4749-57.

23. Xiong $\mathrm{Y}$, Ren $\mathrm{C}$, Zhang B, et al. Analyzing the behavior of a porous nano-hydroxyapatite/polyamide66 (n-HA/PA66) composite for healing of bone defects. Int J Nanomed. 2014. doi:10. 2147/IJN.S52990.

24. Qiao B, Li J, Zhu Q, et al. Bone plate composed of a ternary nano-hydroxyapatite/polyamide66/glass fiber composite: biomechanical properties and biocompatibility. Int J Nanomed. 2014. doi:10.2147/IJN.S57353.

25. Yang X, Song Y, Liu L, et al. Anterior reconstruction with nanohydroxyapatite/polyamide-66 cage after thoracic and lumbar corpectomy. Orthopedics. 2012;35(1):e66-73.

26. Zhao Z, Jiang D, Ou Y, et al. A hollow cylindrical nano-hydroxyapatite/polyamide composite strut for cervical reconstruction after cervical corpectomy. J Clin Neurosci. 2012;19(4):536-40.

27. Gercek E, Arlet V, Delisle J, et al. Subsidence of stand-alone cervical cages in anterior interbody fusion: warning. Eur Spine J. 2003;12(5):513-6.
28. Gruskay JA, Webb ML, Grauer JN. Methods of evaluating lumbar and cervical fusion. Spine J. 2014;14(3):531-9.

29. Pannell WC, Savin DD, Scott TP, et al. Trends in the surgical treatment of lumbar spine disease in the United States. Spine J. 2015;15(8):1719-27.

30. Deyo RA. Fusion surgery for lumbar degenerative disc disease: still more questions than answers. Spine J. 2015;15(2):272-4.

31. Schomacher M, Finger T, Koeppen D, et al. Application of titanium and polyetheretherketone cages in the treatment of pyogenic spondylodiscitis. Clin Neurol Neurosurg. 2014. doi:10. 1016/j.clineuro.2014.09.027.

32. Tokuhashi Y, Ajiro Y, Umezawa N. Subsidence of metal interbody cage after posterior lumbar interbody fusion with pedicle screw fixation. Orthopedics. 2009;32(4):259-64.

33. Choi JY, Sung KH. Subsidence after anterior lumbar interbody fusion using paired stand-alone rectangular cages. Eur Spine J. 2006;15(1):16-22.

34. Xu Q, Lu H, Zhang J, et al. Tissue engineering scaffold material of porous nanohydroxyapatite/polyamide 66. Int J Nanomed. 2010;13(5):331-5 